\title{
Generalized Legendre-Stirling Numbers
}

\author{
K. C. Garrett ${ }^{1}$, Kendra Killpatrick ${ }^{2 *}$ \\ ${ }^{1}$ Department of Mathematics, Statistics and Computer Science, St. Olaf College, Northfield, MN, USA \\ ${ }^{2}$ Natural Science Division, Pepperdine University, Malibu, CA, USA \\ Email: ${ }^{*}$ Kendra.Killpatrick@pepperdine.edu
}

Received 12 August 2014; revised 10 September 2014; accepted 9 October 2014

Copyright (C) 2014 by authors and Scientific Research Publishing Inc.

This work is licensed under the Creative Commons Attribution International License (CC BY).

http://creativecommons.org/licenses/by/4.0/

(c) $\underset{\mathrm{EY}}{0}$ Open Access

\section{Abstract}

The Legendre-Stirling numbers were discovered by Everitt, Littlejohn and Wellman in 2002 in a study of the spectral theory of powers of the classical second-order Legendre differential operator. In 2008, Andrews and Littlejohn gave a combinatorial interpretation of these numbers in terms of set partitions. In 2012, Mongelli noticed that both the Jacobi-Stirling and the Legendre-Stirling numbers are in fact specializations of certain elementary and complete symmetric functions and used this observation to give a combinatorial interpretation for the generalized Legendre-Stirling numbers. In this paper we provide a second combinatorial interpretation for the generalized Legendre-Stirling numbers which more directly generalizes the definition of Andrews and Littlejohn and give a combinatorial bijection between our interpretation and the Mongelli interpretation. We then utilize our interpretation to prove a number of new identities for the generalized LegendreStirling numbers.

\section{Keywords}

Stirling Numbers, Legendre-Stirling Numbers, Set Partitions

\section{Introduction}

The Stirling numbers of the second kind have long played an important role in many areas of combinatorics and have a nice combinatorial interpretation in terms of set partitions (for example, see [1]). For a good discussion of the Stirling numbers of the second kind, the interested reader can reference Stanley's book on Enumerative Combinatorics [2] and Mansour's book on Combinatorics of Set Partitions [1]. In 2002, a generalization of the Stirling numbers of the second kind, called the Legendre-Stirling numbers, was given by Everitt, Littlejohn and Wellman [3]. They discovered these numbers as a result of the study of the classic second-order Legendre differential equation.

\footnotetext{
${ }^{*}$ Corresponding author.
} 


$$
l_{k}[y](x):=-\left(\left(1-x^{2}\right) y^{\prime}(x)\right)^{\prime}+k y(x)=\lambda y(x) \quad(x \in(-1,1))
$$

In 2008, Andrews and Littlejohn [4] found a combinatorial interpretation of the Legendre-Stirling numbers in terms of certain generalized set partitions. In 2012, Mongelli [5] used a specialization of certain symmetric functions to develop a combinatorial interpretation of generalized Legendre-Stirling numbers that doesn't appear to directly generalize the combinatorial interpretation for Andrews and Littlejohn's Legendre-Stirling numbers.

In this paper, we build on Andrews and Littlejohn's combinatorial interpretation of the Legendre-Stirling numbers to give a second combinatorial interpretation of these numbers.

This paper is organized as follows. In Section 2 we review the basic combinatorics related to the Stirling numbers of the second kind. We also review the Legendre-Stirling numbers and then discuss both the combinatorial interpretation given by Andrews and Littlejohn and the combinatorial interpretation of the generalized Legendre-Stirling numbers given by Mongelli. In Section 3, we give our combinatorial interpretation for the generalized Legendre-Stirling numbers using colored set partitions, which is based on the interpretation of Andrews and Littlejohn for Legendre-Stirling numbers. We give an explicit bijection between our interpretation and the Mongelli interpretation to show that these are equivalent. In the final section, we use our combinatorial interpretation to state and prove a number of interesting identities satisfied by the generalized Legendre-Stirling numbers.

\section{Background}

Let $[n]$ denote the set $\{1,2, \cdots, n\}$. A set partition of $[n]$ is a partition of $[n]$ into disjoint, non-empty subsets which are called blocks of the set partition. For example, there are 15 set partitions of [4].

$$
\begin{array}{ccc}
1234 & 12-34 & 1-4-23 \\
1-234 & 13-24 & 2-3-14 \\
2-134 & 14-23 & 2-4-13 \\
3-124 & 1-2-34 & 3-4-12 \\
4-123 & 1-3-24 & 1-2-3-4
\end{array}
$$

Definition 2.1 The number of set partitions of $[n]$ into $k$ blocks is called the Stirling number of the second kind and is denoted by $S(n, k)$.

From the above example, one can see that $S(4,1)=1, S(4,2)=7, S(4,3)=6$ and $S(4,4)=1$. It should be noted that several authors use the notation $\left\{\begin{array}{l}n \\ k\end{array}\right\}$ to refer to the Stirling number of the second kind [6].

The Legendre-Stirling numbers were first defined in 2002 as the coefficients of integral composite powers of the Legendre expression in Lagrangian symmetric form. Andrews and Littlejohn [4] proved that these LegendreStirling numbers satisfy the following slightly more general recurrence relation:

$$
\operatorname{LS}(n, k)=\operatorname{LS}(n-1, k-1)+k(k+1) \operatorname{LS}(n-1, k)
$$

In that same paper, Andrews and Littlejohn gave a combinatorial interpretation of the Legendre-Stirling numbers in the following manner. Let $[\bar{n}]=\{1, \overline{1}, 2, \overline{2}, \cdots, n, \bar{n}\}$, ordered $1<\overline{1}<2<\overline{2}<\cdots<n<\bar{n}$. We will call $i$ and $\bar{i}$ a pair. Then $\operatorname{PS}(n, k)$ is the number of set partitions of $[\bar{n}]$ into $k+1$ blocks with the following restrictions:

1) One block, called the zero block is distinguishable from the others and is the only block that may be empty. The zero block may not contain an element and its pair.

2) The other $k$ blocks are indistinguishable, each must be non-empty, and the smallest two elements of each block must form a pair, while no other two elements in a block may form a pair.

In 2012, Mongelli [5] noted that the Legendre-Stirling numbers are a specialization of certain symmetric functions and gave the following combinatorial interpretation of these numbers for any fixed polynomial with only integer roots and nonnegative coefficients, $f=\left(x+a_{1}\right)\left(x+a_{2}\right) \cdots\left(x+a_{r}\right), a_{1} \leq a_{2} \leq \cdots \leq a_{r}$, and for fixed $j, n, k \in \mathbb{N}$. 
Consider $r$ labeled copies of the numbers $1, \cdots, n$, i.e.

$$
1_{1}, 1_{2}, \cdots, 1_{r}, 2_{1}, 2_{2}, \cdots, 2_{r}, \cdots, n_{1}, n_{2}, \cdots, n_{r}
$$

Consider a pair $P=\left(\pi,\left(S_{1}, \cdots, S_{a_{r}}\right)\right)$ where $\pi$ is a set partition of a subset of $\left\{1_{1}, \cdots, n_{r}\right\}$ into $j$ blocks and $S_{1}, \cdots, S_{a_{r}}$ are subsets of $\left\{1_{1}, \cdots, n_{r}\right\}$. We say that $P$ is $f$-Stirling of order $(n, j)$ if $P$ is a partition of the set of $r$ labeled copies of the numbers 1 through $n$ into $j+a_{r}$ subsets such that

1) The subsets in $\pi$ are nonempty and each one contains the minimum number with all its indices;

2) One of the subsets in $\pi$ contains $1_{1}, 1_{2}, \cdots, 1_{r}$;

3) Each $m_{i}, \quad(1 \leq m \leq n, 1 \leq i \leq r)$ is in one of the first $j+a_{i}$ subsets.

If we specify $f=x(x+1)(x+2) \cdots(x+j-1)$ so that $a_{1}=0, a_{2}=1, \cdots, a_{j}=j-1$, then we define the Mongelli generalized Legendre-Stirling numbers $\operatorname{MLS}_{r}(n, j)$ as the number of set partitions satisfying the above conditions.

Mongelli notes that when $r=2$, so $f=x(x+1)$, there is a straightforward bijection between his combinatorial interpretation and that of Andrews and Littlejohn.

\section{Generalized Legendre-Stirling Numbers, $\operatorname{LS}_{r}(n, j)$}

At the time of publication of the Mongelli paper, the authors of this paper had developed a second combinatorial interpretation of the generalized Legendre-Stirling numbers and used this second interpretation to prove a number of generalized combinatorial identities for these numbers. In this section we give this second combinatorial interpretation for the Legendre-Stirling numbers and describe a bijection between the Mongelli interpretation and this second interpretation (which, as it turns out, is not a generalization of the bijection that Mongelli gives for the special case of $r=2$ ). In the following section, we use this new interpretation to prove several new combinatorial identities for the generalized Legendre-Stirling numbers.

To begin, we define $[n]_{r}=\left\{1_{1}, 1_{2}, \cdots, 1_{r}, 2_{1}, 2_{2}, \cdots, 2_{j}, \cdots, n_{1}, n_{2}, \cdots, n_{r}\right\}$, ordered $1_{1}<1_{2}<\cdots<1_{r}<2_{1}<\cdots<n_{1}<n_{2}<\cdots<n_{r}$. One can think of this set as a set of $r$ colors of the elements 1 through $n$.

Definition 3.1 The generalized Legendre-Stirling numbers, $\operatorname{LS}_{r}(n, j)$, are the number of set partitions of $[n]_{r}$ into $j+r-1$ blocks with the following restrictions:

1) There are $r-1$ distinguishable zero blocks which may be empty and which may contain no more than one color of any element.

2) The remaining $j$ nonzero blocks are indistinguishable, must be non-empty and must contain all $r$ colors of the smallest element in the block. No more than one color of any other element is allowed in these blocks.

Theorem 3.2 $\operatorname{MLS}_{r}(n, j)=\operatorname{LS}_{r}(n, j)$.

Proof. We will show that our combinatorial interpretation of $\operatorname{LS}_{r}(n, j)$ is equivalent to the Mongelli interpretation by giving a bijection between the set partitions described in each interpretation. Recall $f=x(x+1)(x+2) \cdots(x+j-1)$ so $a_{1}=0, a_{2}=1, \cdots, a_{j}=j-1$. We first note that the subsets in $\pi$ in the Mongelli interpretation correspond to the $j$ nonzero blocks in our interpretation. In both interpretations, each of these subsets must be nonempty and must contain the minimum number with all its indices. In the Mongelli interpretation, one of the subsets in $\pi$ must contain $1_{1}, \cdots, 1_{r}$. In our interpretation, since there are $r-1$ zero blocks, at least one color of 1 is contained in one of the $j$ nonzero blocks, thus all colors of 1 must occur in this nonzero block.

The conditions that we need to show are equivalent are condition 3 of the Mongelli description (that each $m_{i}$, $(1 \leq m \leq n, 1 \leq i \leq r)$ is in one of the first $j+(i-1)$ subsets) and our condition that no more than one color of any element is allowed to be in the same block, other than the minimum element in the nonzero blocks.

Let $P$ be a set partition that satisfies the Mongelli conditions with $f=x(x+1)(x+2) \cdots(x+j-1)$. Order the subsets in $\pi$ in decreasing order from left to right, according to the minimal element in the set. To create a set partition $Q$ that satisfies our criterion, place the minimal elements in the same subsets as in $P$. For each $m$ which is not a minimal element in one of the subsets of $\pi$, let $l$ be the subset containing $m_{r}$, counting 
from left to right. Then in $Q$, place $m_{r}$ in the $(r+j-l)$ th available block, counting from right to left, where a block is considered available if it does not already contain any labeled copies of $m$. Repeat the process for $m_{r-1}$, then $m_{r-2}$ and so on until all labeled copies of $m$ have been placed. Do this for all $m$ that are not the minimal element in one of the subsets of $\pi$. The result will be a set partition in which none of the colors of $m$, for $m$ not a minimal element in one of the zero blocks, is contained in a block with another color of $m$, thus giving us a set partition $Q$ which satisfies our criterion.

To see that this procedure is a bijection, we can create the inverse map. Begin with a set partition $Q$ that satisfies our criteria. We will order the blocks by putting the $j$ nonzero blocks first, ordered in decreasing order of minimal element, followed by the zero blocks. To form $P$, place the minimal elements in the same blocks as in $Q$. For $m$ not a minimal element in a nonzero block, begin with $m_{r}$. Let $l$ be the number of blocks to the right of the block containing $m_{r}$ that either do not contain a labeled copy of $m$ or contain an $m_{i}$ with $i<r$. Place $m_{r}$ into the $(r+1-l)$ th block of $P$, counting from left to right. Repeat the process for $m_{r-1}$, then $m_{r-2}$ and so on until all labeled copies of $m$ have been placed. Do this for all $m$ that are not the minimal element in one of the nonzero blocks.

Example 3.3 Let $n=3, \quad r=5$ and $j=2$ and suppose we have the following Mongelli set partition

$$
P=\left\{\left\{2_{1}, 2_{2}, 2_{3}, 2_{4}, 2_{5}, 3_{5}\right\},\left\{1_{1}, 1_{2}, 1_{3}, 1_{4}, 1_{5}, 3_{1}, 3_{2}, 3_{4}\right\} \mid\left\{3_{3}\right\}, \varnothing, \varnothing, \varnothing\right\}
$$

To form $Q$, we will place the 1's and the 2's in the same blocks as $P$. Then for $3_{5}$ we have $l=1$, for $3_{4}$ we have $l=2$, for $3_{3}$ we have $l=3$, for $3_{2}$ we have $l=2$ and for $3_{1}$ we have $l=2$. Thus our resulting $Q$ partition that satisfies our criteria is

$$
Q=\left\{\left\{2_{1}, 2_{2}, 2_{3}, 2_{4}, 2_{5}, 3_{5}\right\},\left\{1_{1}, 1_{2}, 1_{3}, 1_{4}, 1_{5}\right\} \mid\left\{3_{4}\right\},\left\{3_{2}\right\},\left\{3_{3}\right\},\left\{3_{1}\right\}\right\}
$$

\section{Identities for $\operatorname{LS}_{r}(n, j)$}

The Stirling numbers of the second kind satisfy a number of interesting identities which can be found nicely stated in Benjamin and Quinn's book [6]. Our combinatorial interpretation of the generalized Legendre-Stirling numbers suggests several analogous identities which we state and prove below. We put the number of the identity from the book that is being generalized in parentheses in the following theorems. In several of the proofs in the section, we use the abbreviation LME to refer to the largest element which is a minimum in its block. We will also utilize the notation of Stanley [2] that $(x)_{k}=x(x-1)(x-2) \cdots(x-k+1)$.

Theorem 4.1 (201) Let $m, n$ be non-negative integers with $n \geq m$ and let $r$ be a positive integer. Then,

$$
\sum_{k=m+1}^{n+1} \operatorname{LS}_{r}(k-1, m)\left[(m+r)_{r}\right]^{n+1-k}=\operatorname{LS}_{r}(n+1, m+1)
$$

Proof. The right hand side counts the number of partitions of $[n+1]_{r}$ into $(m+1)$ nonzero blocks and $r-1$ zero blocks. For the left hand side, let $k$ be the largest minimum element that appears with its $r$-tuple in a block all by itself. Then the elements 1 through $k-1$ can be distributed into the remaining $m$ nonzero blocks and $r-1$ zero blocks in $\operatorname{LS}_{r}(k-1, m)$ ways. The elements $k+1$ through $n+1$ must be distributed into the $r-1$ zero blocks and the $m+1$ nonzero blocks so that none of the $r$ colors of the same element appear together. Since the $m+1$ nonzero blocks all contain smaller elements at this point, they can now be considered distinguishable. Thus we can begin by first distributing the $r$ colors of $k+1$, then the $r$ colors of $k+2$, etc. To distribute the $r$ colors of $k+1$, there are $(m+1)+(r-1)=m+r$ choices for the block to place $(k+1)_{1}$ in, $m+r-1$ choices to place $(k+1)_{2}$ in, and so on. This is accounted for by $(m+r)_{r}$. Since we must do this for each of the remaining $n+1-k$ elements, this is a total of $\left[(m+r)_{r}\right]^{n+1-k}$.

Theorem 4.2 (199) Let $m, n$ be non-negative integers and let $r$ be a positive integer. Then,

$$
\mathrm{LS}_{r}(m+n+1, m)=\sum_{k=0}^{m}(k+r-1)_{r} \operatorname{LS}_{r}(n+k, k)
$$

Proof. The left hand side of this identity counts the ways to distribute the elements of the set $[m+n+1]_{r}$ into $m+r-1$ blocks with $m$ nonzero blocks. For the right hand side, consider the ways to distribute the elements 
of the set $[m+n+1]_{r}$ into $m+r-1$ blocks with $m$ nonzero blocks where the elements $m+n+1, m+n$, $m+n-1, \cdots, n+k+2$ each appear in a block with its $r$-tuple and nothing else and the element $n+k+1$ does not appear in a block with its $r$-tuple. To count the ways to create a distribution like this, first place the elements $m+n+1$ through $n+k+2$ in nonzero blocks alone with their $r$-tuples. This leaves $m-((m+n+1)-(n+k+2)+1)=k$ nonzero blocks for the remaining elements. Since $n+k+1$ does not appear in a block with its $r$-tuple, the $r$ colors of this element must each appear in a different block which can be counted by $(k+r-1)(k+r-2) \cdots(k+1) k=(k+r-1)_{r}$. The remaining $n+k$ elements are distributed into the $k$ nonzero blocks and the $r-1$ zero blocks with the usual restrictions, which can be counted by $\operatorname{LS}_{r}(n+k, k)$.

Theorem 4.3 (208) Let $m, n$ be non-negative integers with $n \geq m$ and let $r$ be a positive integer. Then, $\sum_{i=m+1}^{n+1} \sum_{k=0}^{n+1-i} \operatorname{LS}_{r}(i-1, m)\left(\begin{array}{c}n+1-i \\ k\end{array}\right) r^{k}\left[(m+r-1)_{r-1}\right]^{n+1-i} \times(m+r)^{n+1-i-k}(-1)^{k}=\sum_{i=m+1}^{n+1} \operatorname{LS}_{r}(i-1, m)\left[(m+r-1)_{r}\right]^{n+1-i}$

Proof. We will show that both sides count the ways to partition $[n+1]_{r}$ into $(m+1)+(r-1)=m+r$ blocks with the LME in a block by itself. To begin, we look at the right hand side. Let $i$ be the LME and let it appear in a block by itself, i.e. with only its $r$-tuple and no other elements. Then partition the $i-1$ smaller elements into the remaining $m+r-1$ blocks in $\operatorname{LS}_{r}(i-1, m)$ ways. The remaining $n+1-i$ larger elements must then be distributed to $m+r-1$ blocks since they can be in any block except the block with $i$. In addition, all $r$ colors of a given element must appear in different blocks. Thus for a given element $k$, there are $m+r-1$ choices of block for the first color of $k, m+r-2$ choices of block for the second color of $k$ and so on, giving $(m+r-1)_{r}$ ways to distribute the $r$ different colors of the element $k$. Since there are $n+1-i$ elements distributed in this way, this contributes $\left[(m+r-1)_{r}\right]^{n+1-i}$ to the sum. Finally, since the LME can range from $m+1$ to $n+1$ we get the sum on the right hand side.

Since the left hand side has an alternating sign in the summation, we will describe what the terms count and then give a sign reversing involution on the terms that will leave us with only the partitions of $[n+1]_{r}$ into $(m+r)$ blocks with the LME in a block by itself. Again we will let $i$ be the LME in any of the $m+1$ regular blocks. We partition the $i-1$ smaller elements into the remaining $m+r-1$ blocks in $\operatorname{LS}_{r}(i-1, m)$ ways. Next choose $k$ elements from the remaining $n+1-i$ elements to be put in the block with $i$ and to be considered barred elements. For each of these $k$ elements we have $r$ choices of which color element is included giving us a total term of $\left(\begin{array}{c}n+1-i \\ k\end{array}\right) r^{k}$. We then have $r-1$ colors of each of these $k$ elements to be distributed into the other $m+r-1$ blocks so that no two of the same type are in the same block. This can be done in $\left[(m+r-1)_{r-1}\right]^{k}$ ways. The remaining $n+1-i-k$ elements can be distributed into any of the $m+r$ blocks as long as no two elements of the same type appear in the same block. If any of these elements are in the block with $i$ they are considered to be unbarred elements. This distribution can be done in $\left[(m+r)_{r}\right]^{n+1-i-k}$ ways. Summing over possible choices of $k$ and $i$ gives us the summation on the left hand side. The $(-1)^{k}$ term gives a -1 for every barred element in the block containing $i$.

For the sign-reversing involution, let $l>i$ be the smallest element in the block containing $i$, if such an element exists. If $l$ is barred, remove the bar. If $l$ is unbarred, add a bar. This changes the sign on the corresponding term and gives a sign-reversing involution. The fixed points are those partitions with $i$ in a block by itself.

\section{References}

[1] Mansour, T. (2012) Combinatorics of Set Partitions. Discrete Mathematics and Its Applications Series, Chapman and 
Hall/CRC an Imprint of Taylor and Francis LLC.

[2] Stanley, R. (2012) Enumerative Combinatorics. In Cambridge Studies in Advanced Mathematics, Cambridge University Press, Cambridge, Vol. 49.

[3] Everitt, W.N., Littlejohn, L.L. and Wellman, R. (2002) Legendre Polynomials, Legendre-Stirling Numbers, and the Left-Definite Spectral Analysis of the Legendre Differential Expression. Journal of Computational and Applied Mathematics, 148, 213-238. http://dx.doi.org/10.1016/S0377-0427(02)00582-4

[4] Andrews, G.E. and Littlejohn, L.L. (2009) A Combinatorial Interpretation of the Legendre-Stirling Numbers. Proceedings of the American Mathematical Society, 137, 2581-2590.

[5] Mongelli, P. (2012) Combinatorial Interpretations of Particular Evaluations of Complete and Elementary Symmetric Functions. Electronic Journal of Combinatorics, 19, \#P60.

[6] Benjamin, A. and Quinn, J. (2003) Proofs That Really Count: The Art of Combinatorial Proof. Mathematical Association of America, Providence, RI. 
Scientific Research Publishing (SCIRP) is one of the largest Open Access journal publishers. It is currently publishing more than 200 open access, online, peer-reviewed journals covering a wide range of academic disciplines. SCIRP serves the worldwide academic communities and contributes to the progress and application of science with its publication.

Other selected journals from SCIRP are listed as below. Submit your manuscript to us via either submit@scirp.org or Online Submission Portal.
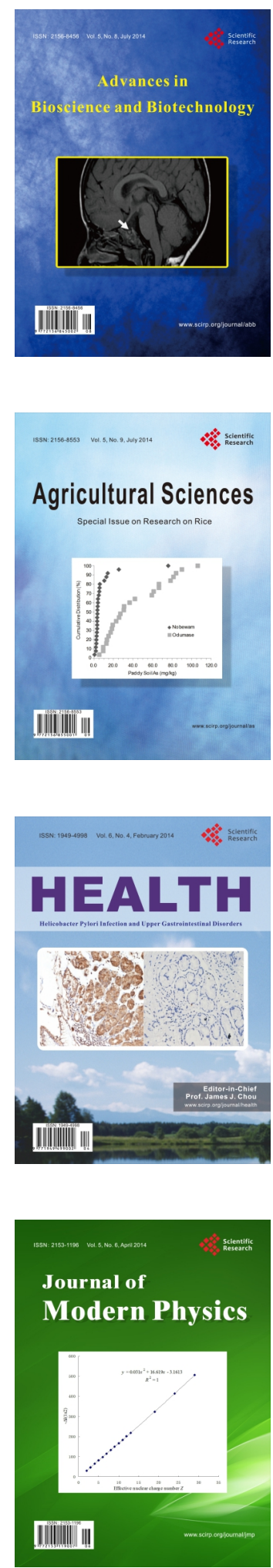
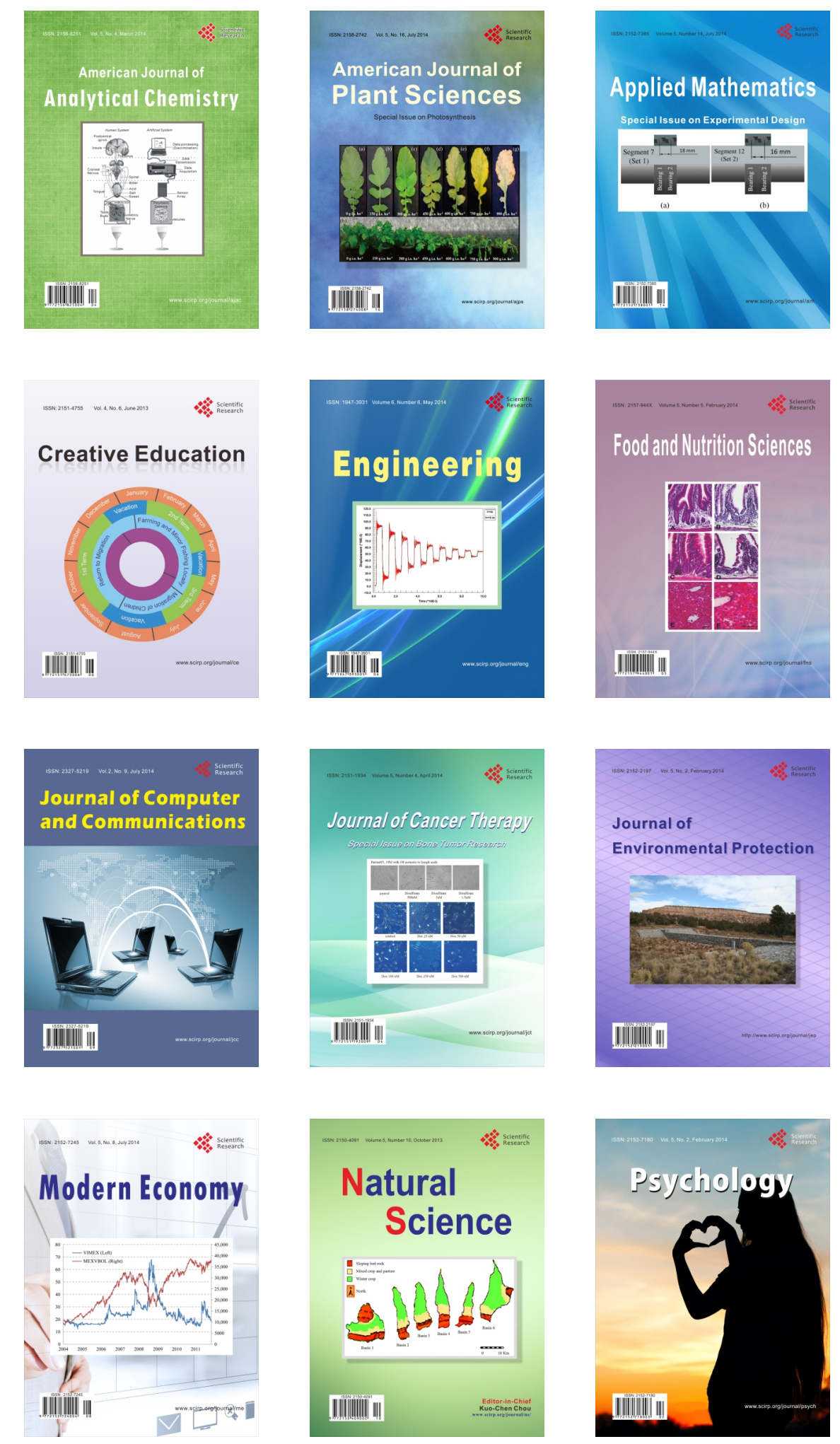